\title{
Intrinsically patterned two-dimensional materials for selective adsorption of molecules and nanoclusters
}

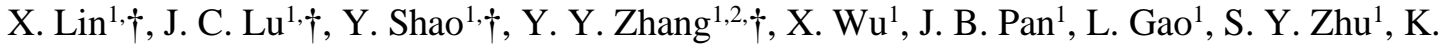

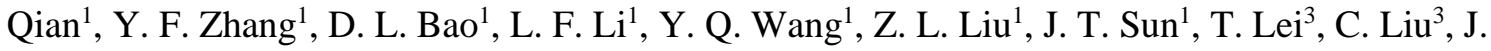 \\ O. Wang ${ }^{3}$, K. Ibrahim ${ }^{3}$, D. N. Leonard ${ }^{4}$, W. Zhou ${ }^{1,4}$, H. M. Guo ${ }^{1}$, Y. L. Wang ${ }^{1, *}$, S. X. Du ${ }^{1, *}$, S. \\ T. Pantelides ${ }^{2,1}$, H.-J. Gao ${ }^{1, *}$ \\ 1. Institute of Physics \& University of Chinese Academy of Sciences, Chinese Academy of \\ Sciences, Beijing 100190, P. R. China \\ 2. Department of Physics and Astronomy and Department of Electrical Engineering and \\ Computer Science, Vanderbilt University, Nashville, Tennessee 37235, USA \\ 3. Institute of High Energy Physics, Chinese Academy of Sciences, Beijing 100049, P. R. China \\ 4. Material Sciences and Technology Division, Oak Ridge National Laboratory, Oak Ridge, \\ Tennessee 37381, USA
}

$\dagger$ These authors contributed equally to this work and are listed alphabetically.

*Corresponding author. E-mail: ylwang@iphy.ac.cn (Y.L.W); sxdu@iphy.ac.cn (S.X.D); hjgao@iphy.ac.cn (H.J.G)

Two-dimensional (2D) materials have been studied extensively as monolayers ${ }^{1-5}$, vertical or lateral heterostructures ${ }^{6-8}$. To achieve functionalization, monolayers are often patterned using soft lithography and selectively decorated with molecules ${ }^{\mathbf{9}, 10}$. Here we demonstrate the growth of a family of 2D materials that are intrinsically patterned. We demonstrate that a monolayer of PtSe2 can be grown on a Pt substrate in the form of a triangular pattern of alternating $1 \mathrm{~T}$ and $1 \mathrm{H}$ phases. Moreover, we show that, in a monolayer of CuSe grown on a $\mathrm{Cu}$ substrate, strain relaxation leads to periodic patterns of triangular nanopores with uniform size. Adsorption of different species at preferred pattern sites is also achieved, demonstrating that these materials can serve as templates for selective self-assembly of molecules or nanoclusters, as well as for the functionalization of the same substrate with two different species. 
Two-dimensional (2D) materials have unique and unusual properties that promise novel applications $6,11,12$. Typically, 2D materials need to be functionalized and patterned, just as semiconductor films need to be doped $n$-type or $p$-type in a lithographically-patterned way to produce pn junctions (light-emitting diodes, junction lasers, resistors) and transistor structures 10,11 . Functionalization of $2 \mathrm{D}$ materials is done in a variety of ways, e.g., by doping with impurities or molecules, either within the 2D lattices or adsorbed on the exposed surfaces ${ }^{10}$. In particular, functionalization by adsorbates can facilitate catalysis, sensing, optical or magnetic response, nanodevices, or other applications. To achieve selective or multiple functionalization by adsorbates, patterning by soft lithography has been used ${ }^{9} .2 \mathrm{D}$ materials exhibiting forms of atomic-scale patterning have been proposed based on calculations by the choice of Bravais lattice ${ }^{13}$ or stoichiometry ${ }^{14,15}$. Moiré patterns represent a nano-scale form of patterning imposed by the substrate on otherwise homogeneous $2 \mathrm{D}$ materials ${ }^{16,17}$.

Herein, we report the fabrication of prototypes of a family of $2 \mathrm{D}$ materials that are intrinsically patterned on the nano-scale and can be selectively or dually functionalized by adsorbates. We report two prototype 2D materials, each obtained by direct selenization of a monatomic metal substrate. More specifically, we show that, by controlling the annealing temperature and deposition amount of Se on a Pt substrate, we obtain either a homogeneous monolayer $\mathrm{PtSe}_{2}$ in the $1 \mathrm{~T}$ phase or a "patterned monolayer" comprising a periodic triangular structure of alternating $1 \mathrm{H}$ and $1 \mathrm{~T}$ phases. The size of the triangles can be tuned by varying the density of deposited Se atoms. The choice between a homogeneous $1 \mathrm{~T}$ phase or patterned $1 \mathrm{H} / 1 \mathrm{~T}$ phases is reversible. We also report the fabrication of a patterned monolayer of $\mathrm{CuSe}$ having periodic arrays of triangular nanopores, with distinct domains. In both patterned materials, adsorption of different species at preferred pattern components has been achieved, illustrating the potential for selective/dual functionalization. In the above two prototype intrinsically-patterned monolayers, we show that $\mathrm{Fe}_{13} \mathrm{Se}_{7}$ nanoclusters, a known catalyst ${ }^{18}$, can be grown selectively in the nanopores of patterned $\mathrm{CuSe}$ monolayer, providing a way to avoid aggregation in nanoparticle catalysts. Another molecular species, iron(II) phthalocyanine (FePc), can be selectively adsorbed on the $\mathrm{CuSe}$ 
surface between the nanopores. Throughout the exposition we use density-functionaltheory (DFT) calculations to account for the observations.

A prototype of a 2D intrinsically-patterned material is shown schematically in Fig. 1a. The entire monolayer is $\mathrm{PtSe}_{2}$, but the two distinct types of triangles are two different phases, 1H and 1T (blue and yellow triangles, respectively). A large-scale STM image of such a structure is shown in Fig. 1b. We will describe shortly how the patterned structure was fabricated and how we determined that the two kinds of moiré patterns, exhibiting honeycomb and hexagonal features, are the $1 \mathrm{H}$ and $1 \mathrm{~T}$ phase of $\mathrm{PtSe}_{2}$, respectively. The honeycomb and hexagonal structures are more visible in the intermediate-resolution STM images of Fig. 1c to 1e. These images also demonstrate that triangles of different sizes are possible. Atomic-resolution images are shown in Fig. 1f to 1h, together with simulations in Fig. 1i to 1k. The detail structures are shown in Supplementary Information Fig. S1 to S3. A clean, sharp interface is clearly visible in Fig. 1g.

The formation of a homogeneous monolayer $1 \mathrm{~T}-\mathrm{PtSe}_{2}$ on a $\mathrm{Pt}(111)$ substrate by direct selenization has been described in Ref. 19. Such a monolayer, fabricated by annealing at $270^{\circ} \mathrm{C}$ during the selenization of a Pt substrate is shown in Fig. 2a. A homogeneous 1T sample transforms to a triangular pattern (Fig. 2b) by annealing at $400^{\circ} \mathrm{C}$. This elevated temperature leads to the loss of some selenium, leaving vacancies (marked by the yellow arrow) in the film. The transformation process is monitored by the change of chemical states of Se atoms via in-situ X-ray photoelectron spectroscopy (XPS) measurements. As shown in Fig. 2c, the $\mathrm{Se} 3 d$ core level from the pure 1T structure $\mathrm{PtSe}_{2}$ monolayer exhibits two peaks (colored in red). After annealing the sample to $400^{\circ} \mathrm{C}$, the intensity of the initial red peaks decreases and two new peaks (colored in blue) appear. The new peaks have an energy shift of $0.4-\mathrm{eV}$ relative to the initial ones. Considering that a similar energy shift in the XPS spectra has been reported between $\mathrm{H}$ - and T-phases in $\mathrm{MoTe}_{2}$ flakes ${ }^{11}$, the XPS energy shift (Fig. 2c) in $\mathrm{PtSe}_{2}$ is consistent with a local 1T-to-1H transformation. Theoretical calculations indicate that free-standing $1 \mathrm{H}-\mathrm{PtSe}_{2}$ monolayer is not stable (Supplementary Information Fig. S4). For the patterned structure with alternating triangular $1 \mathrm{~T}$ and $1 \mathrm{H}$ areas, the $1 \mathrm{H}$ domains are limited in size and are likely stabilized by the surrounding $1 \mathrm{~T}-\mathrm{PtSe}_{2}$. 
We also measured I(z) spectra, which provide information on the local work function. As shown in Supplementary Information Fig. S5, the derived work function in 1T domains is larger than that in $1 \mathrm{H}$ domains, which is confirmed by DFT calculations. These results provide further evidence of the existence of $1 \mathrm{H}-\mathrm{PtSe}_{2}$.

The transformation of a pure 1T phase to a patterned 1H/1T phase is reversible. By adding Se atoms and annealing the sample to $\sim 270^{\circ} \mathrm{C}$, the triangular pattern reverts to a defectfree homogeneous 1T structure (Fig. 2a). The extra peaks in the XPS spectrum of the patterned 1H/1T phase (blue peaks in Fig. 2c) also disappear, confirming that the sample reverts to a pure 1T structure. The disappearance of the Se vacancies during the reversible transformation provides evidence that Se vacancies mediate the formation of $1 \mathrm{H}$ domains.

Based on the above experimental data, we use the schematic drawings in Fig. $2 \mathrm{~d}$ to $\mathrm{g}$, to show the transformation process and mechanism. Specifically, thermal annealing of a pristine 1T structure $\mathrm{PtSe}_{2}$ film at an elevated temperature leads to the loss of top-layer $\mathrm{Se}$ atoms (depicted by the arrows in Fig. 2b), generating Se vacancies (Fig. 2e). DFT calculations find that lines of vacancies are energetically preferred (see Supplementary Information Fig. S6) which is in agreement with a previous report ${ }^{20}$. Because of the threefold point symmetry, three directions of such lines are possible, which leads to the formation of triangles, as shown in Fig. $2 \mathrm{f}$. Within the triangle surrounded by vacancies, a transformation of the $1 \mathrm{~T}$ phase to the $1 \mathrm{H}$ phase occurs and leads to a $1 \mathrm{H} / 1 \mathrm{~T}$ patterned structure. A similar structural transformation has been reported in a $\mathrm{MoS}_{2}$ monolayer ${ }^{21}$. More details of the 1T-to-1H transformation in $\mathrm{PtSe}_{2}$ can be found in Supplementary Information Fig. S6 and S7.

An alternative method to produce the $1 \mathrm{H} / 1 \mathrm{~T}$ triangular pattern is to control the initial density of Se atoms. This process is shown schematically in Supplementary Information Fig. S8. Whereas for the fabrication of a homogenous $1 \mathrm{~T}-\mathrm{PtSe}_{2}$, the crucible is filled with the Se source and held at-center for a long time to deliver ample amounts of Se everywhere on the sample. While the $1 \mathrm{H} / 1 \mathrm{~T}$ triangular pattern is fabricated by holding the crucible filled with the Se source off-centered to the substrate, resulting in a Se density gradient, which subsequently leads to different structures. A triangular tiling pattern of $1 \mathrm{H} / 1 \mathrm{~T}-\mathrm{PtSe} \mathrm{S}_{2}$ 
forms in the area with a relatively low Se density. As the density of Se atoms increases, the size of $1 \mathrm{~T}-\mathrm{PtSe}_{2}$ triangles increases and the number of $1 \mathrm{H}-\mathrm{PtSe} \mathrm{e}_{2}$ triangles decreases significantly. As the Se density increases further, it eventually leads to a defect-free homogeneous 1T-PtSe 2 film. An intermediate structure can be found in between the 1H/1Ttiling region and the homogeneous $1 \mathrm{~T}$ region. Typical STM images with different Se amounts are shown in Supplementary Information Fig. S8. The XPS mapping of Se $3 d$ electrons along the direction of increasing Se density areas (area (1) to area (4)) confirms a significant decrease of $1 \mathrm{H}-\mathrm{PtSe}_{2}$ and increase of $1 \mathrm{~T}-\mathrm{PtSe}_{2}$. These data confirm the role of Se vacancies and suggest that a critical concentration is necessary to produce a periodic triangular pattern.

The highly-ordered triangular tiling patterns constructed by $1 \mathrm{H}$ and $1 \mathrm{~T}$ phases of a monolayer $\mathrm{PtSe}_{2}$ make it a suitable template for selective adsorption of different species. Figures $2 \mathrm{~h}$ and $2 \mathrm{i}$ show a large-scale area and zoomed-in images of pentacene $\left(\mathrm{C}_{22} \mathrm{H}_{14}\right)$ on patterned $\mathrm{PtSe}_{2}$. It is clear that pentacene molecules adsorb selectively on $1 \mathrm{H}$ triangles, allowing the formation of a periodic array of molecules. The high-resolution STM image shown in Fig. 2i demonstrates more details for the nanoclusters, which present three orientations corresponding to the triangular shape of the $1 \mathrm{H}-\mathrm{PtSe}_{2}$ region.

The preference of molecules to bind on the $1 \mathrm{~T}$ or $1 \mathrm{H}$ domain is determined by the relative binding energies. As shown in Table S1, all the tested molecules have larger binding energies on the $1 \mathrm{H}$ domain. The basic idea, nevertheless, is that different molecules can in principle adsorb on the two phases of an intrinsically-patterned 2D material, leading to simultaneous dual functionalization for catalysis or other applications. The possibility of different objects adsorbing on different regions of a patterned material will be demonstrated in the next prototype intrinsically-patterned 2D material, namely monolayer $\mathrm{CuSe}$ on a $\mathrm{Cu}$ substrate.

In addition to $1 \mathrm{H} / 1 \mathrm{~T}$ patterned $\mathrm{PtSe}_{2}$, we have fabricated an intrinsically-patterned form of monolayer $\mathrm{CuSe}$ by direct selenization of $\mathrm{Cu}(111)$ substrate (more details of the fabrication process are given in Supplementary Information). A large-scale STM image of a monolayer $\mathrm{CuSe}$ with periodic arrays of identical triangular nanopores is shown in Fig. 3a. The 
nanopores form a hexagonal lattice as indicated by the green hexagonal lattice matrix with a periodicity of $\sim 3 \mathrm{~nm}$. The figure clearly shows domains with triangular nanopores of opposite orientations, separated by domain boundaries comprising parallelogram-shaped nanopores. High-resolution STM images of a domain-boundary region and a single nanopore are shown in Fig. $3 \mathrm{~b}$ and 3c, respectively. The shape of the nanopores, honeycomb lattice of $\mathrm{CuSe}$, and the zigzag edges of the nanopore are clearly resolved.

An atomic-resolution STM image of a patterned monolayer CuSe with nanopores is shown in Fig. 3d. Based on a low-energy-electron-diffraction (LEED) pattern (as shown in Supplementary Information Fig. S9), a $(4 \sqrt{ } 3 \times 4 \sqrt{ } 3)$ CuSe sitting on a $(11 \times 11) \mathrm{Cu}(111)$ model is proposed. The optimized atomic configuration is shown in Fig. 3f. For better visualization, the $\mathrm{Cu}(111)$ substrate is hidden. The simulated STM image based on the proposed atomic model is shown in Fig. 3e, in excellent agreement with the experimental data. Note that the wiggly atomic lines (marked by purple curves in Fig. 3d to f) induced by the release of stress are well reproduced by the DFT simulations.

To further confirm that the fabricated CuSe is a monolayer, we performed a cross-section high-angle annular-dark-field (HAADF) scanning transmission electron microscopy (STEM) study. An experimental image, a simulated image and side view of the structure are shown in Fig. 3, g to i, respectively. The excellent agreement further proves that the $\mathrm{CuSe}$ pattern with periodic nanopores is a monolayer material.

The periodicity and size of the nanopores can be explained as follows. In principle, the $\mathrm{Cu}$ substrate and a CuSe monolayer are incommensurate. The two lattices can be made commensurate, however, if a $(4 \sqrt{3} \times 4 \sqrt{3})$ CuSe supercell is stretched by $1.5 \%$. This supercell then registers on a $(11 \times 11) \mathrm{Cu}(111)$ surface supercell, with a central $\mathrm{Cu}$ atom of the monolayer supercell on top of a $\mathrm{Cu}$ atom on the substrate. Removal of this central atom and three shells of atoms around it, a total of 13 atoms, produces the observed nanopores at the observed periodicity. In Supplementary Information Fig. S10, we demonstrate that removal of these 13 atoms amounts to removing three complete hexagonal rings and corresponds to maximum gain of energy (negative formation energy) compared with other 
options for removing atoms. We conclude that formation of 13-atom nanopores at the observed periodicity is the energetically preferred way to relieve the lattice-mismatch strain.

We have been able to demonstrate selective adsorption on the patterned $\mathrm{CuSe}$ monolayer with nanopores. Fe atoms deposited on a CuSe monolayer form W-shape clusters in the nanopore hexagonal superstructure as shown in the large-scale STM image in Fig. 4a. Considering the threefold symmetry of a nanopore, three different orientations of W-shape clusters can be found, as indicated by three "W" letters in Fig. 4a. DFT calculations suggest that the $\mathrm{W}$-shape cluster contains $13 \mathrm{Fe}$ atoms (the atomic configuration and a discussion about other possibilities are presented in Supplementary Information Fig. S11). Simulation of the STM image based on this model is provided in Fig. 4c, showing excellent agreement with the atomic-resolution STM image (Fig. 4b). The proposed model is further confirmed by in-situ XPS measurements. As shown in Fig. $4 d$, the Fe $2 p_{3 / 2}$ and Fe $2 p_{1 / 2}$ core-level spectra are located at $707.24 \mathrm{eV}$ and $720.40 \mathrm{eV}$, respectively, which are similar to the $\mathrm{Fe}$ $2 p$ core levels in bulk $\mathrm{FeSe}^{22}$. In addition to the $\mathrm{W}$-shape clusters in the periodic nanopores, zigzag-shaped clusters also form in the more extended parallelogram-shaped nanopores at domain boundaries (see Supplementary Information Fig. S12).

In addition to the selective adsorption in nanopores, additional selective functionalization is achieved by deposition FePc molecules on the terraces. An atomic model of FePc is shown in the inset of Fig. 4f. As shown in Fig. 4e, when we deposit FePc molecules onto a patterned monolayer $\mathrm{CuSe}$, the molecules are monodispersed and adsorbed only on $\mathrm{CuSe}$ terraces. A zoomed-in STM image (Fig. 4f) shows a clear cross feature, which is a typical apparent topography of FePc molecules ${ }^{23}$. It is noticed that FePc molecules on a $\mathrm{CuSe}$ substrate have preferred orientations. The angles between FePc molecules (indicated by the sketched purple cross) and the $\mathrm{CuSe}[1 \overline{1} 0]$ direction (white line) is $\sim 45^{\circ}$. As a result of the threefold symmetry of the substrate and the fourfold symmetry of the molecule, there are three equivalent preferred orientations as indicated by the three purple crosses in Fig. $4 \mathrm{e}$.

Comparing the two intrinsically-patterned monolayers, i.e. 1H/1T-PtSe 2 and $\mathrm{CuSe}$ with periodic nanopores, they are both achieved by direct selenization. After annealing at a high 
temperature, monolayer $1 \mathrm{~T}-\mathrm{PtSe}_{2}$ undergoes a phase transition and forms the $1 \mathrm{H} / 1 \mathrm{~T}$ pattern, while periodic nanopores appear in $\mathrm{CuSe}$ monolayer. We attribute the difference in the two prototype systems to the interactions between the epitaxial monolayer and the substrate. In the $\mathrm{PtSe} / \mathrm{Pt}(111)$ system, the van der Waals interaction between $\mathrm{PtSe}_{2}$ and the Pt substrate gives freedom to flip the structure from $1 \mathrm{~T}$ to $1 \mathrm{H}$. In contrast, the interaction between $\mathrm{CuSe}$ monolayer and a $\mathrm{Cu}$ substrate is much stronger. Emission of atoms and formation of nanopores becomes the way to lower the energy.

Both the $\mathrm{PtSe}_{2}$ and $\mathrm{CuSe}$ samples are stable in ambient conditions. The samples were removed from the high-vacuum chamber and were kept in air without protection for more than 12 hours. The samples were then put back to the high-vacuum chamber and annealed at $200{ }^{\circ} \mathrm{C}$ for $\mathrm{PtSe}_{2}$ and $400{ }^{\circ} \mathrm{C}$ for $\mathrm{CuSe}$ to remove the possible adsorbates. STM images show that the samples keep their patterns (see Supplementary Information Fig. S13). The stability in air makes these patterned monolayers proper for potential applications involving ambient conditions.

In summary, we demonstrate a concept of intrinsically-patterned $2 \mathrm{D}$ materials which can be selectively functionalized. We demonstrate the fabrication of patterned 2D monolayers in two cases: $1 \mathrm{H} / 1 \mathrm{~T}$ monolayer $\mathrm{PtSe}_{2}$ tiling pattern and monolayer $\mathrm{CuSe}$ with periodic nanopores. Selective adsorptions are achieved in both cases. This work ushers a frontier for the fabrication of intrinsically patterned 2D materials.

\section{Acknowledgements}

We acknowledge the financial support from National Key Research \& Development Projects of China (2016YFA0202300), the National Basic Research Program of China (2013CBA01600), the National Natural Science Foundation of China (Nos. 61390501, 51572290, 61306015 and 61471337, 51325204) and the Chinese Academy of Sciences (Nos. 1731300500015, XDB07030100, and the CAS Pioneer Hundred Talents Program). A portion of the research was performed in CAS Key Laboratory of Vacuum Physics. Work at Vanderbilt (STP and YYZ) was supported by the U.S. Department of Energy under grant 
DE-FG02-09ER46554 and by the McMinn Endowment. Computations by YYZ were carried out at the National Energy Research Scientific Computing Center, a DOE Office of Science User Facility supported by the Office of Science of the U.S. Department of Energy under Contract No. DE-AC02-05CH11231. The electron microscopy work was supported in part by the U.S. Department of Energy, Office of Science, Basic Energy Science, Materials Sciences and Engineering Division, and through a user project at ORNL's Center for Nanophase Materials Sciences, which is a DOE Office of Science User Facility.

\section{Author contributions}

H.J.G. and S.T.P. conceived and coordinated the research project. X.L. designed the CuSe experiments. J.C.L. and K.Q. prepared CuSe samples and performed the STM experiments. Y.L.W. designed the PtSe 2 experiments. Y.S., X.W., S.Y.Z., L.F.L., Y.Q.W., Z.L.L., and H.M.G. prepared PtSe $\mathrm{P}_{2}$ samples and performed the STM experiments. T.L., C.L., J.O.W. and K.I. provided supports for XPS experiments. D.N.L. and W.Z. performed the STEM experiments. Y.Y.Z., J.B.P., L.G., Y.F.Z., D.L.B., and J.T.S performed the DFT calculations under the guidance of S.X.D. All authors participated in discussing the data and editing the manuscript.

\section{Additional information}

Supplementary information is available in the online version of the paper. Reprints and permissions information is available online at www.nature.com/reprints. Correspondence and requests for materials should be addressed to Y.L.W., S.X.D., or H.-J.G.

\section{Competing financial interests}

The authors declare no competing financial interests. 


\section{References}

[1] Novoselov, K. S. et al. A roadmap for graphene. Nature 490, 192-200 (2012).

[2] Oughaddou, H. et al. Silicene, a promising new 2D material. Prog. Surf. Sci. 90, 46-83 (2015).

[3] Desai, S. B. et al. $\mathrm{MoS}_{2}$ transistors with 1-nanometer gate lengths. Science 354, 99-102 (2016).

[4] Chhowalla, M. et al. The chemistry of two-dimensional layered transition metal dichalcogenide nanosheets. Nat. Chem. 5, 263-275 (2013).

[5] Yin, K. et al. Unsupported single-atom-thick copper oxide monolayers. 2D Mater. 4, 011001 (2017).

[6] Geim, A. K. \& Grigorieva, I. V. Van der Waals heterostructures. Nature 499, 419-425 (2013).

[7] Eda, G. et al. Coherent atomic and electronic heterostructures of single-layer $\mathrm{MoS}_{2}$. ACS Nano 6, 7311-7317 (2012).

[8] Withers, F. et al. Light-emitting diodes by band-structure engineering in van der Waals heterostructures. Nat. Mater. 14, 301-306 (2015).

[9] Jung, M. W. et al. Novel fabrication of flexible graphene-based chemical sensors with heaters using soft lithographic patterning method. ACS Appl. Mater.

Interfaces 6, 13319-13323 (2014).

[10] Joshi, S. et al. Control of molecular organization and energy level alignment by an electronically nanopatterned boron nitride template. ACS Nano 8, 430-442 (2014).

[11] Cho, S. et al. Phase patterning for ohmic homojunction contact in MoTe2. Science 349, 625-628 (2015).

[12] Voiry, D. et al. The role of electronic coupling between substrate and 2D $\mathrm{MoS}_{2}$ nanosheets in electrocatalytic production of hydrogen. Nat. Mater. 15, 1003-1009 (2016).

[13] Pan, L. D., Zhang, L. Z., Song, B. Q., Du, S. X. \& Gao, H.-J. Graphyne- and graphdiyne-based nanoribbons: density functional theory calculations of electronic structures. Appl. Phys. Lett. 98, 173102 (2011).

[14] Gao, G., Jiao, Y., Waclawik, E. R. \& Du, A. Single atom (Pd/Pt) supported on graphitic carbon nitride as an efficient photocatalyst for visible-light reduction of carbon dioxide. J. Am. Chem. Soc. 138, 6292-6297 (2016). 
[15] Zhang, H., Li, Y., Hou, J., Tu, K. \& Chen, Z. FeB 6 monolayers: the graphene-like material with hypercoordinate transition metal. J. Am. Chem. Soc. 138, 5644-5651 (2016).

[16] Pan, Y. et al. Highly ordered, millimeter-scale, continuous, single-crystalline graphene monolayers formed on Ru(0001). Adv. Mater. 21, 2777-2780 (2009).

[17] Lee, M. et al. Ballistic miniband conduction in a graphene superlattice. Science 353, 1526-1529 (2016).

[18] Huang, S. et al. Ultrathin $\mathrm{FeSe}_{2}$ nanosheets: controlled synthesis and application as a heterogeneous catalyst in dye-sensitized solar cells. Chem. Eur. J 21, 40854091 (2015).

[19] Wang, Y. et al. Monolayer PtSe 2 , a new semiconducting transition-metaldichalcogenide, epitaxially grown by direct selenization of Pt. Nano Lett. 15, 4013-4018 (2015).

[20] Lin, J., Pantelides, S. T. \& Zhou, W. Vacancy-induced formation and growth of inversion domains in transition-metal dichalcogenide monolayer. ACS Nano $\mathbf{9}$, 5189-5197 (2015).

[21] Lin, Y. C., Dumcenco, D. O., Huang, Y. S. \& Suenaga, K. Atomic mechanism of the semiconducting-to-metallic phase transition in single-layered $\mathrm{MoS}_{2}$. Nat. Nanotech. 9, 391-396 (2014).

[22] Yamasaki, A. et al. Electron correlation in the FeSe superconductor studied by bulk-sensitive photoemission spectroscopy. Phys. Rev. B 82, 184511 (2010).

[23] Cheng, Z. H. et al. High resolution scanning-tunneling-microscopy imaging of individual molecular orbitals by eliminating the effect of surface charge. Surf. Sci. 605, 415-418 (2011). 


\section{Methods:}

Preparation of 1H/1T PtSe 2 on Pt(111) substrate by transforming from 1T PtSe2. Single-layer $1 \mathrm{~T} \mathrm{PtSe}_{2}$ films were grown in a commercial ultrahigh vacuum (UHV) system (Omicron), with a base pressure better than $3 \times 10^{-10}$ mbar, equipped with standard MBE capabilities. The $\operatorname{Pt}(111)$ substrate was cleaned by several cycles of argon-ion sputtering followed by annealing until sharp $(1 \times 1)$ diffraction spots in the LEED pattern and clean surface terraces in the STM images were obtained. High-purity Se (99.99\%, Sigma-Aldrich) evaporated from a Knudsen cell was deposited onto the clean $\operatorname{Pt}(111)$ surface at room temperature. The sample was subsequently annealed up to $270^{\circ} \mathrm{C}$ to achieve selenization and crystallization, which leads to homogeneous $1 \mathrm{~T} \mathrm{PtSe} \mathrm{P}_{2}$ films on $\mathrm{Pt}(111)$ substrate. By annealing this sample at $400^{\circ} \mathrm{C}$, the $1 \mathrm{~T} \mathrm{PtSe}_{2}$ transforms to the $1 \mathrm{H} / 1 \mathrm{~T} \mathrm{PtSe}_{2}$ with triangular patterns.

Preparation of 1H/1T PtSe on $\operatorname{Pt}(111)$ substrate by controlling the Se initial density. As shown in Supplementary Information Fig. S4, an alternative method to produce the $1 \mathrm{H} / 1 \mathrm{~T}$ triangular pattern is to control the Se initial density. Having obtained the clean $\operatorname{Pt}(111)$ surface, the crucible filled with the Se source was aligned at the edge instead of the center of the substrate during the fabrication process. This off-center alignment leads to varying initial density of Se atoms at different regions of the substrate, resulting in a $\mathrm{Se}$ density gradient. The sample was subsequently annealed up to $270^{\circ} \mathrm{C}$ to achieve selenization and crystallization, which finally leads to different structures. A triangular tiling pattern of $1 \mathrm{H} / 1 \mathrm{~T} \mathrm{PtSe} \mathrm{P}_{2}$ forms in the area with a relative low initial Se density. As the density of Se atoms increases, the size of $1 \mathrm{~T} \mathrm{PtSe} \mathrm{P}_{2}$ triangles increases and the number of $1 \mathrm{H} \mathrm{PtSe} e_{2}$ triangles decreases significantly. As the Se density increases further, it finally leads to a defect-free homogeneous $1 \mathrm{~T} \mathrm{PtSe}_{2}$ film.

Preparation of patterned $\mathrm{CuSe}$, Fe/CuSe and $\mathrm{FePc} / \mathrm{CuSe}$ on $\mathrm{Cu}(111)$ substrate. The growth of $2 \mathrm{D}$ patterned CuSe was carried out in a commercial ultrahigh vacuum (UHV) system (Omicron) (base pressure better than $1 \times 10^{-10}$ mbar) equipped with a sample preparation chambers. Clean single-crystal $\mathrm{Cu}(111)$ (MaTecK) surface was obtained via cycles of argon-ion sputtering followed by annealing at $550^{\circ} \mathrm{C}$. The cleanliness of the $\mathrm{Cu}(111)$ surface was verified by STM scanning. The 2D patterned CuSe sample was obtained by Se $(99.99 \%$, Sigma-Aldrich) deposition from a standard Knudsen cell while the $\mathrm{Cu}$ substrate was held at room temperature, and post-annealing at $200^{\circ} \mathrm{C}$. For the $\mathrm{Fe} / \mathrm{CuSe}$ and $\mathrm{FePc} / \mathrm{CuSe}$ samples, the beam of Fe (99.99\%, ESPI) and the FePc molecules (99.99\%, Sigma-Aldrich) were generated from a commercially available e-beam cell 
(Omicron) and a standard Knudsen cell, respectively. During the deposition, the patterned $\mathrm{CuSe}$ sample was kept at room temperature. After preparation, the samples were transferred to the Low-Temperature-STM head operating at $\sim 4 \mathrm{~K}$.

Ex-situ STEM characterization of CuSe on Cu(111) substrate. Cross-section TEM sample preparation. A layer of amorphous carbon of $\sim 100 \mathrm{~nm}$ in thickness was first deposited onto the as-grown $\mathrm{CuSe}$ monolayer on a $\mathrm{Cu}$ substrate shortly after removal from the MBE chamber. A TEM cross-section sample was prepared along the $\mathrm{Cu}$ [112] zone axis using focused ion beam (FIB) and was further thinned to $40 \mathrm{~nm}$ thick using low-energy ion milling. STEM-ADF (scanning transmission electron microscopy - annular dark field) imaging. The experimental STEM-ADF imaging was performed on an aberration corrected Nion UltraSTEM-100 operated at $100 \mathrm{kV}$. The convergence semi-angle was set to $30 \mathrm{mrad}$, and the ADF images were collected from 80-200 mrad semi-angle range. The thickness of the cross-section sample was measured to be $\sim 40 \mathrm{~nm}$ using electron energy loss spectroscopy. STEM-ADF image simulation was performed using QSTEM with the same accelerating voltage, probe-forming angle and detector angle as those used in experiment. A single-crystal $\mathrm{CuSe}$ domain was used in the simulation, with the thickness of the crosssection sample set to $22 \mathrm{~nm}$.

STM and LEED measurements of the as-grown samples. STM images of the samples were acquired in the constant-current mode, using an electrochemically etched tungsten tip. All voltages were applied to the sample with respect to the tip. The Nanotec Electronica WSxM software was used to process the STM images. Low-energy electron diffraction (LEED) was employed with a 4-grid detector (Omicron Spectra LEED) in a connected UHV chamber to identify the superstructure macroscopically.

XPS measurements of the as-grown samples. The in situ X-ray photoelectron spectroscopy (XPS) measurements of the samples were conducted at the Beijing Synchrotron Radiation Facility (BSRF). After growth, the samples were transferred by a UHV suitcase to the XPS station for measuring the change of chemical states of elements. The monochromatic synchrotron radiation is realized by four high-resolution gratings and is controlled by a hemispherical energy analyzer. The photon energy is in the range from 10 to $1100 \mathrm{eV}$.

Theoretical calculations. Quantum mechanical calculations based on density functional theory (DFT) are performed using the Vienna ab initio simulation package (VASP) ${ }^{24,25}$. The projector augmented wave method is employed to describe the core electrons. The local density approximation (LDA) ${ }^{26,27}$ is used for exchange and correlation. The 
rotationally invariant $\mathrm{LDA}+\mathrm{U}$ formalism proposed by Dudarev et al. is used ${ }^{28}$ and $\mathrm{U}_{\text {eff }}$ is chosen as $6.52 \mathrm{eV}$ and $4.3 \mathrm{eV}$ for $\mathrm{Cu}$ and Fe respectively ${ }^{29}$. The electronic wave functions are expanded in plane waves with a kinetic energy cutoff of $400 \mathrm{eV}$. The periodic slab models of $\mathrm{PtSe}_{2} / \mathrm{Pt}(111)$ include four layers of (4×4) $\mathrm{Pt}(111)$ substrate, and monolayer of (3×3) $\mathrm{PtSe}_{2}$. The k-points sampling is $5 \times 5 \times 1$, generated automatically with the origin at the $\Gamma$-point. The periodic slab models of $\mathrm{CuSe} / \mathrm{Cu}(111)$ include three layers of $(11 \times 11)$ $\mathrm{Cu}(111)$ and $(4 \sqrt{3} \times 4 \sqrt{3}) \mathrm{CuSe}$. The k-points sampling is $1 \times 1 \times 1$. For all the calculations, the bottom two layers of substrate atoms are fixed, while all the other atoms are fully relaxed. The structures were relaxed until the energy and residual force on each atom were smaller than $10^{-4} \mathrm{eV}$ and $0.02 \mathrm{eV} / \AA$, respectively. The vacuum layers of the two models

are larger than $15 \AA$. The STM simulations are performed using the Tersoff-Hamann approach $^{30}$.

Data availability. The data that support the findings of this study are available from the corresponding author upon reasonable request.

\section{References:}

[24] Kresse, G. \& Furthmüller, J. Efficiency of ab-initio total energy calculations for metals and semiconductors using a plane-wave basis set. Comput. Mater. Sci. 6, 15-50 (1996).

[25] Kresse, G. \& Furthmüller, J. Efficient iterative schemes for ab initio total-energy calculations using a plane-wave basis set. Phys. Rev. B 54, 11169 (1996).

[26] Ceperley, D. M. \& Alder, B. J. Ground state of the electron gas by a stochastic method. Phys. Rev. Lett. 45, 566-569 (1980).

[27] Perdew, J. P. \& Zunger, A. Self-interaction correction to density-functional approximations for many-electron systems. Phys. Rev. B 23, 5048 (1981).

[28] Dudarev, S. L., Botton, G. A., Savrasov, S. Y., Humphreys, C. J. \& Sutton, A. P. Electron-energy-loss spectra and the structural stability of nickel oxide: an LSDA+U study. Phys. Rev. B 57, 1505 (1998).

[29] Cococcioni, M. \& de Gironcoli, S. Linear response approach to the calculation of the effective interaction parameters in the LDA+U method. Phys. Rev. B 71, 035105 (2005).

[30] Tersoff, J. \& Hamann, D. R. Theory of the scanning tunneling microscope. Phys. Rev. B 31, 805-813 (1985). 

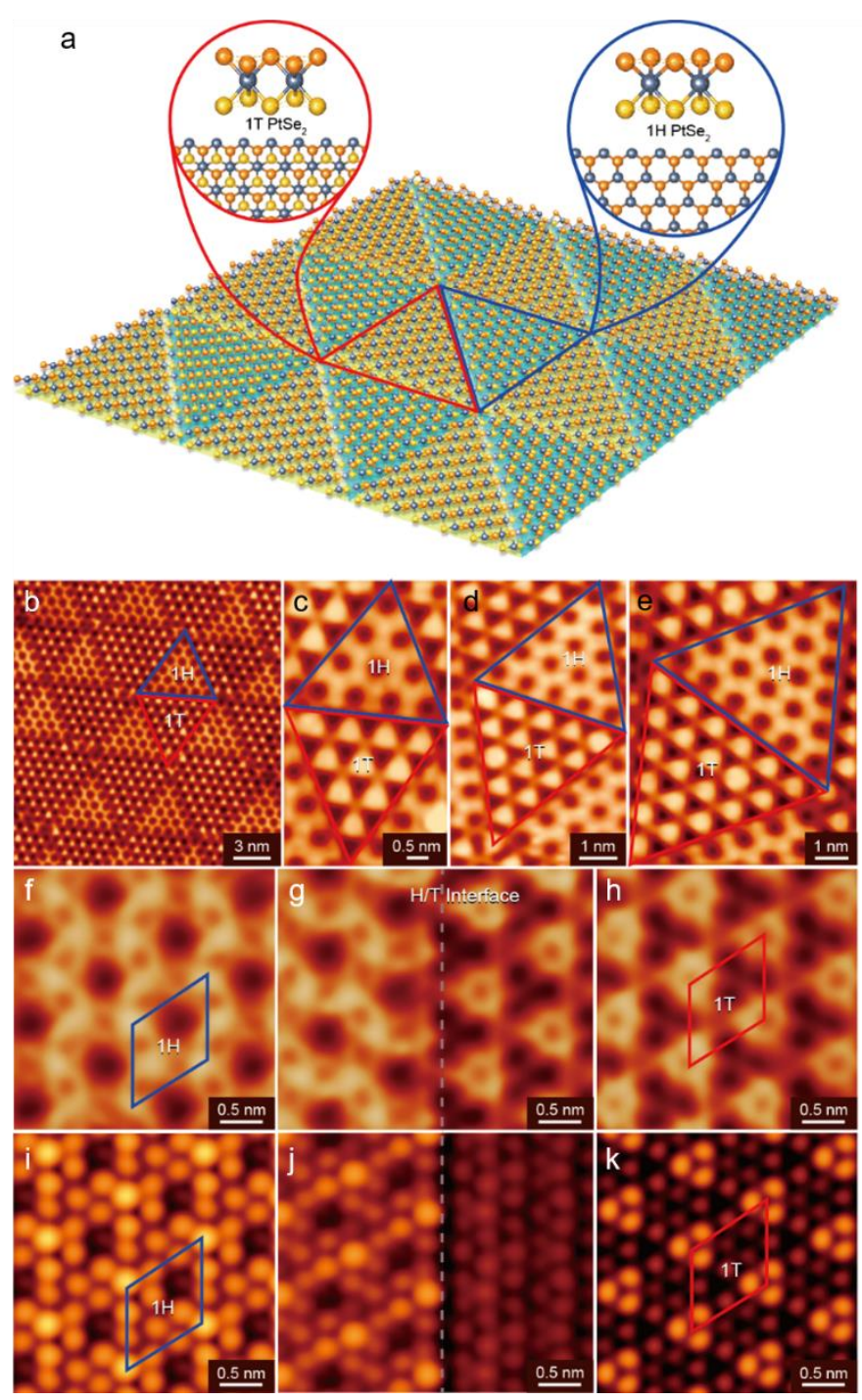

Figure 1 | 1H/1T tiling pattern in monolayer PtSe2. a, Schematic illustration of the triangular tiling pattern formed by alternating $1 \mathrm{H}-$ and $1 \mathrm{~T}-\mathrm{PtSe} 2$ areas, which are colored blue and yellow, respectively. Two views of atomic models for the two configurations are shown in the respective inserts. The top-layer Se atoms, Pt atoms and the bottom-layer Se atoms are colored orange, blue and yellow, respectively. b, STM image of the 1H/1T patterned structure in monolayer $\mathrm{PtSe}_{2} . \mathrm{V}_{\mathrm{s}}=-1.6 \mathrm{~V}, \mathrm{I}_{\mathrm{t}}=0.1 \mathrm{nA}$. c-e, STM images of $1 \mathrm{H}-$ and 1T-PtSe 2 domains of different sizes. $\mathrm{V}_{\mathrm{s}}=-1.6 \mathrm{~V}, \mathrm{I}_{\mathrm{t}}=0.5 \mathrm{nA}$. f-h, High-resolution STM images of $1 \mathrm{H}-\mathrm{PtSe}_{2}(\mathbf{f}), 1 \mathrm{H} / 1 \mathrm{~T}$ interface $(\mathbf{g})$, and $1 \mathrm{~T}-\mathrm{PtSe}_{2}(\mathbf{h})$. The unit cells of the $1 \mathrm{H}$ and $1 \mathrm{~T}$ domains are marked by blue and red diamonds, respectively. $\mathrm{V}_{\mathrm{s}}=-1.0 \mathrm{~V}, \mathrm{I}_{\mathrm{t}}=0.8 \mathrm{nA}$. i-k, STM simulations of a $1 \mathrm{H}-\mathrm{PtSe}_{2}$ domain (i), the 1H/1T interface (j), and a $1 \mathrm{~T}-\mathrm{PtSe} \mathrm{e}_{2}$ domain (k), respectively. 


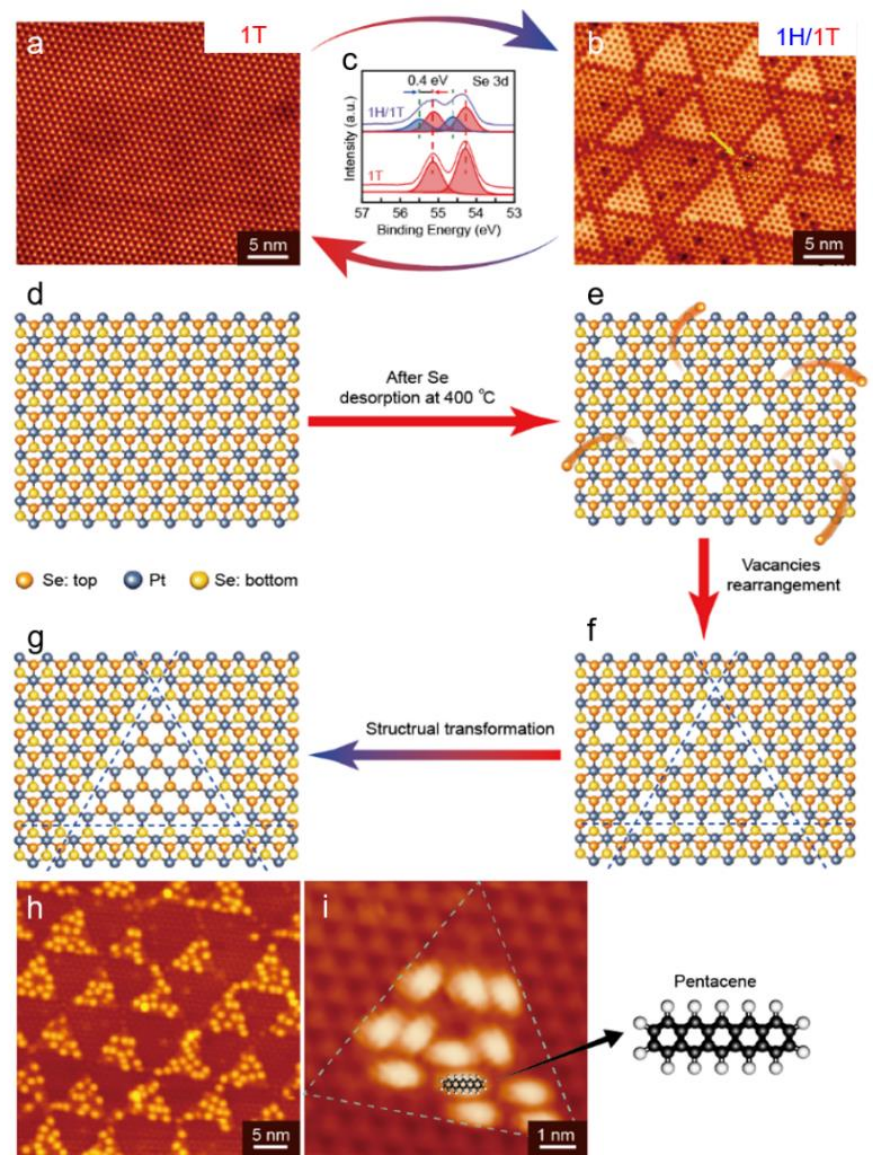

Figure 2 | Reversible transition and selective adsorption of 1H/1T tiling pattern. a and b, Typical STM images of pure 1T-PtSe 2 (a) and 1H/1T patterned $\mathrm{PtSe}_{2}(\mathbf{b})$. The 1H/1T tiling structure can be obtained from a pure $1 \mathrm{~T}$ structure by annealing the sample to $400^{\circ} \mathrm{C}$. The reverse process (from 1H/1T tiling to pure $1 \mathrm{~T}$ structure) can be realized by adding Se and annealing at $270^{\circ} \mathrm{C} . \mathrm{V}_{\mathrm{s}}=-1.6 \mathrm{~V}, \mathrm{I}_{\mathrm{t}}=0.1 \mathrm{nA}$. c, The XPS spectra of Se $3 d$ electrons for the entire reversible structural transformation process. The red and purple peaks originate from Se $3 \mathrm{~d}$ electrons in $1 \mathrm{~T}$ - and $1 \mathrm{H}-\mathrm{PtSe}_{2}$ films with a $0.4-\mathrm{eV}$ shift, respectively. d-g, Schematic illustration of the structural transformation from pure $1 \mathrm{~T}-\mathrm{PtSe}_{2}$ to $1 \mathrm{H} / 1 \mathrm{~T}$ patterned $\mathrm{PtSe}_{2}$. The formation of $1 \mathrm{H}-\mathrm{PtSe}_{2}$ domains is a result of the collective evolution of Se vacancies. h, An STM image of pentacene molecules selectively adsorbed on the 1H$\mathrm{PtSe}_{2}$ domains of a $1 \mathrm{H} / 1 \mathrm{~T}$ patterned $\mathrm{PtSe}_{2}$ film, forming well-ordered triangular clusters. $V_{\mathrm{s}}=-1.6 \mathrm{~V}, \mathrm{I}_{\mathrm{t}}=0.5 \mathrm{nA}$. i, High-resolution STM image of pentacene molecules selectively adsorbed on one $1 \mathrm{H}-\mathrm{PtSe}_{2}$ domain. A schematic of a pentacene molecule is shown on the right. $\mathrm{V}_{\mathrm{s}}=-1.0 \mathrm{~V}, \mathrm{I}=0.8 \mathrm{nA}$. 


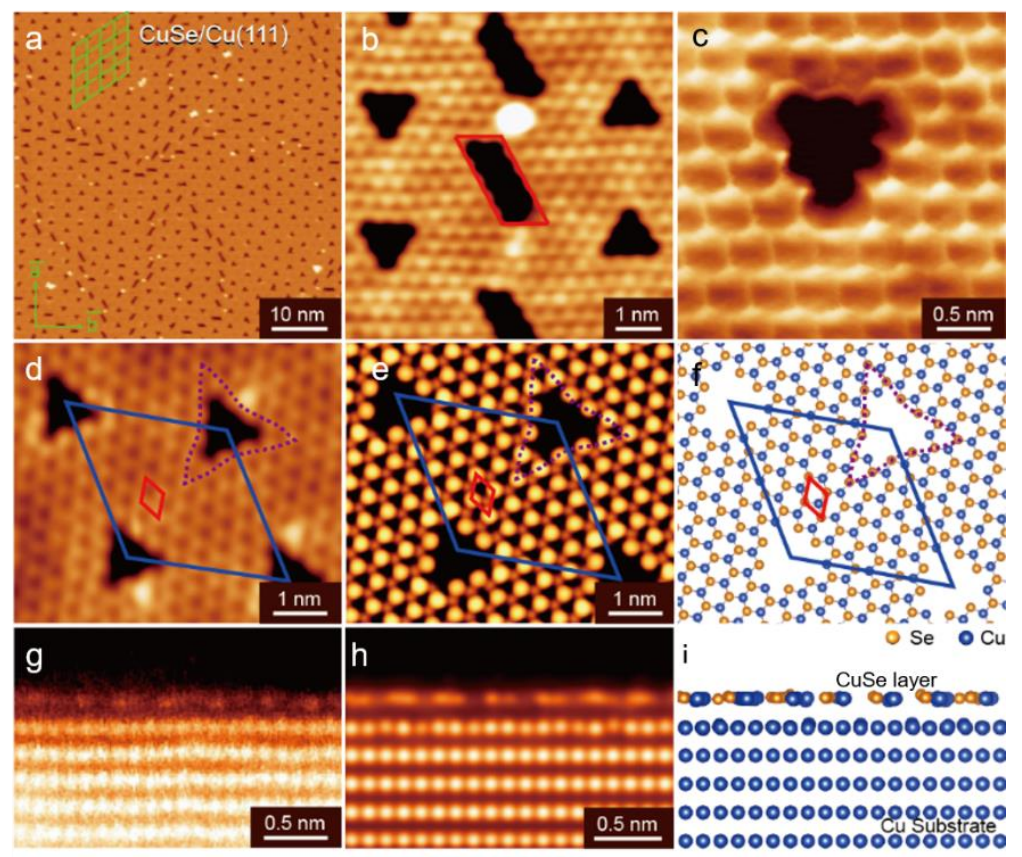

Figure 3 | Patterned CuSe monolayer with periodic nanopores. a, A large-scale STM image of patterned CuSe monolayer. The green lattice presents the 3-nm periodicity of the nanopores. $\mathrm{V}_{\mathrm{s}}=-1.5 \mathrm{~V}, \mathrm{I}_{\mathrm{t}}=0.1 \mathrm{nA}$. b, A high-resolution STM image of a domain boundary region. The boundary consists of parallelogram-shaped nanopores as indicated by the red parallelogram. $\mathrm{V}_{\mathrm{s}}=-1.0 \mathrm{~V}, \mathrm{I}_{\mathrm{t}}=0.3 \mathrm{nA}$. c, A high-resolution STM image of a single nanopore. Honeycomb lattice of $\mathrm{CuSe}$ and the zigzag edges of the nanopore are clearly resolved. $\mathrm{V}_{\mathrm{s}}=-0.01 \mathrm{~V}, \mathrm{I}_{\mathrm{t}}=7.0 \mathrm{nA}$. d-f An atomic-resolution STM image (d), simulated STM image (e), and theoretical model (f) of $\mathrm{CuSe}$ monolayer on $\mathrm{Cu}(111)$ with periodic nanopores, respectively. The images show the detailed structure of nanopores. $\mathrm{V}_{\mathrm{s}}=-1.0 \mathrm{~V}$, $\mathrm{I}_{\mathrm{t}}=0.4 \mathrm{nA}$. In $\mathbf{f}, \mathrm{Cu}$ substrate is hidden for visualization purposes. $\mathbf{g}$-i, Cross-section STEM image (g), simulated cross-section STEM image (h), and side view (i) of a CuSe monolayer on a $\mathrm{Cu}(111)$ substrate. The STEM image confirms that the patterned $\mathrm{CuSe}$ is single layer. 

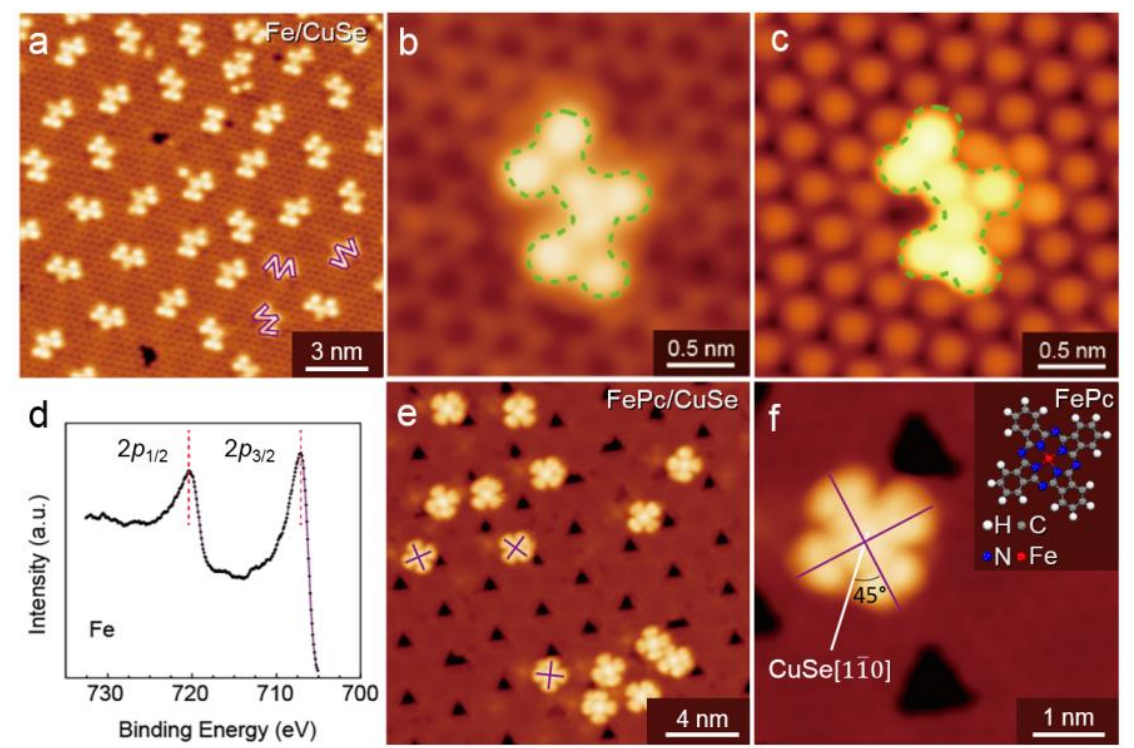

Figure 4 | Selective adsorption on patterned CuSe with periodic nanopores. a, A largescale STM image of CuSe monolayer with W-shaped FeSe clusters. The clusters selectively adsorb in the nanopores of CuSe monolayer. $\mathrm{V}_{\mathrm{s}}=-0.1 \mathrm{~V}, \mathrm{I}_{\mathrm{t}}=1.5 \mathrm{nA}$. b, A highresolution STM image of the $\mathrm{W}$-shaped cluster. The image shows that the $\mathrm{W}$-shaped cluster contains seven protrusions. $\mathrm{V}_{\mathrm{s}}=-0.1 \mathrm{~V}, \mathrm{I}_{\mathrm{t}}=1.5 \mathrm{nA}$. c, A simulated STM image of an $\mathrm{Fe}_{13} \mathrm{Se}_{7}$ cluster in the nanopores. The green contour line and the seven protrusions perfectly match those in (b). d, XPS data showing the Fe $2 p_{3 / 2}$ and Fe $2 p_{1 / 2}$ core levels. e, A largescale STM image of FePc molecules on CuSe. The FePc molecules selectively adsorb on $\mathrm{CuSe}$ terraces with preferential orientations as discussed in the text. $\mathrm{V}_{\mathrm{s}}=-1.1 \mathrm{~V}, \mathrm{I}_{\mathrm{t}}=0.1$ nA. f, High-resolution STM image of an FePc molecule on CuSe. Atomic structure of the FePc molecule is shown in inset. $\mathrm{V}_{\mathrm{s}}=-1.1 \mathrm{~V}, \mathrm{I}_{\mathrm{t}}=1.5 \mathrm{nA}$. 


\section{Supplementary Text and Figures:}

\section{Formation of the moiré pattern.}
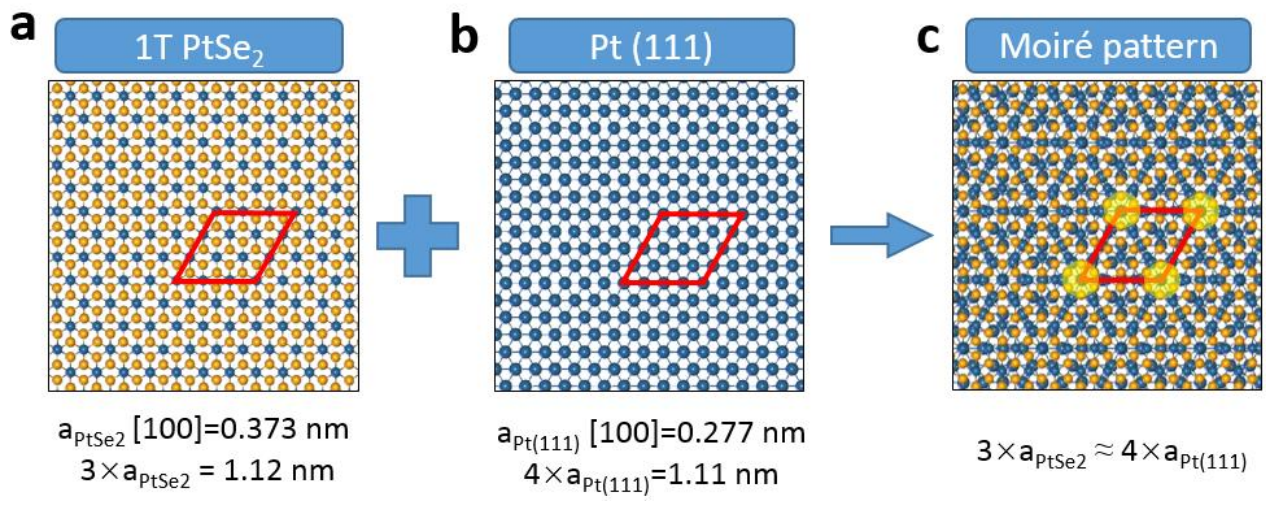

Figure S1 | Models for the origin of the moiré pattern in $\mathrm{PtSe}_{2}$ on $\mathrm{Pt}(111)$. a, A model of the $1 \mathrm{~T} \mathrm{PtSe} 2$ lattice. A (3×3) cell is marked by the red rhombus. $\mathbf{b}$, A model of the Pt(111) lattice. A $(4 \times 4)$ cell is marked by the red rhombus. c, Because the size of a $(3 \times 3) \mathrm{PtSe}_{2}$ approximately equals to that of a (4×4) $\mathrm{Pt}(111)$, when a $\mathrm{PtSe}_{2}$ monolayer sits on a $\mathrm{Pt}(111)$ substrate, moiré pattern forms with a (3×3)PtSe - on-(4×4 $) \operatorname{Pt}(111)$ supercell. 
2. The STM images, relaxed models and similated STM images of 1H- and 1T-PtSe2 on Pt (111).

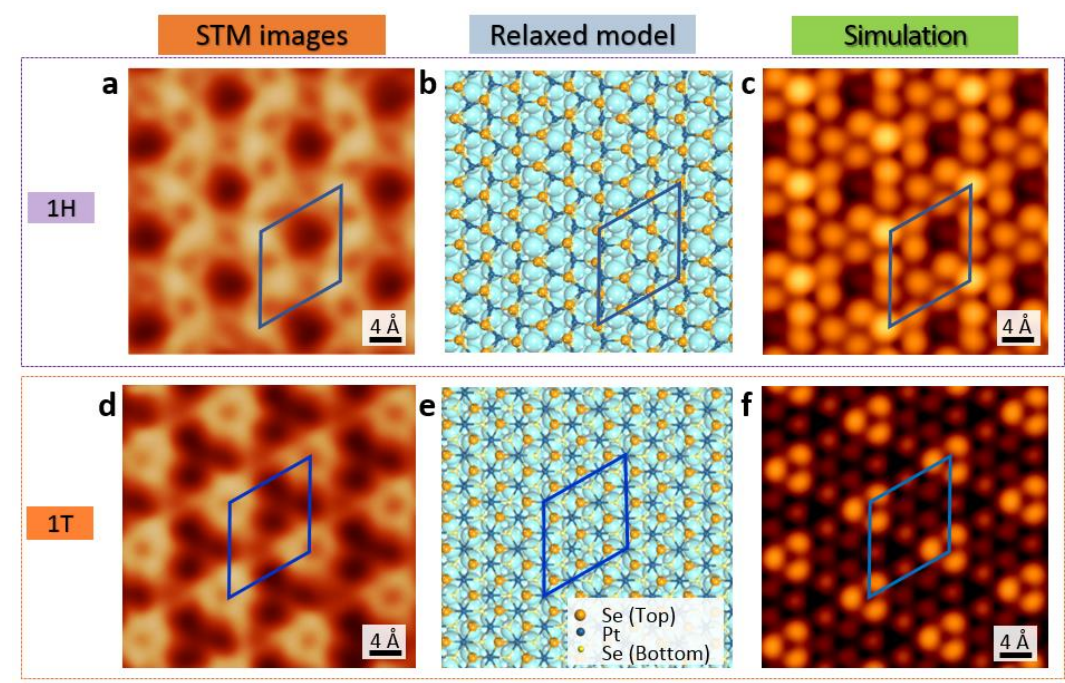

Figure S2 | STM images, relaxed models and simulated STM images of 1H- and 1TPtSe2 on Pt (111). a, STM image for $1 \mathrm{H}-\mathrm{PtSe}_{2}$ on $\mathrm{Pt}(111)$. $\mathrm{V}_{\mathrm{s}}=-1.0 \mathrm{~V}, \mathrm{I}_{\mathrm{t}}=0.8 \mathrm{nA}$. A unit cell of this moiré pattern is marked by the blue diamond. The brightest protrusions at the vertex of diamond are interpreted to be the Se atoms at atop site of Pt (111) surface. b, Top view of the relaxed model for $1 \mathrm{H}-\mathrm{PtSe} \mathrm{S}_{2}$ on $\mathrm{Pt}(111)$. c, $\mathrm{STM}$ simulation for $1 \mathrm{H}-\mathrm{PtSe} 2$ on $\mathrm{Pt}$ (111). The overall features of the experimental STM image (Fig. S2a) are well reproduced. d, STM image for 1T-PtSe 2 on $\mathrm{Pt}(111)$. $\mathrm{V}_{\mathrm{s}}=-1.0 \mathrm{~V}, \mathrm{I}_{\mathrm{t}}=0.8 \mathrm{nA}$. e, Top view of the relaxed model for $1 \mathrm{~T}-\mathrm{PtSe} \mathrm{S}_{2}$ on $\mathrm{Pt}(111)$. f, STM simulation for $1 \mathrm{~T}-\mathrm{PtSe} \mathrm{S}_{2}$ on $\mathrm{Pt}(111)$, which is also in good agreement with the STM image (Fig. S2d). 
3. The tip effect in the STM image simulations of $1 \mathrm{H}-\mathrm{PtSe}_{2}$ on $\operatorname{Pt}(111)$.
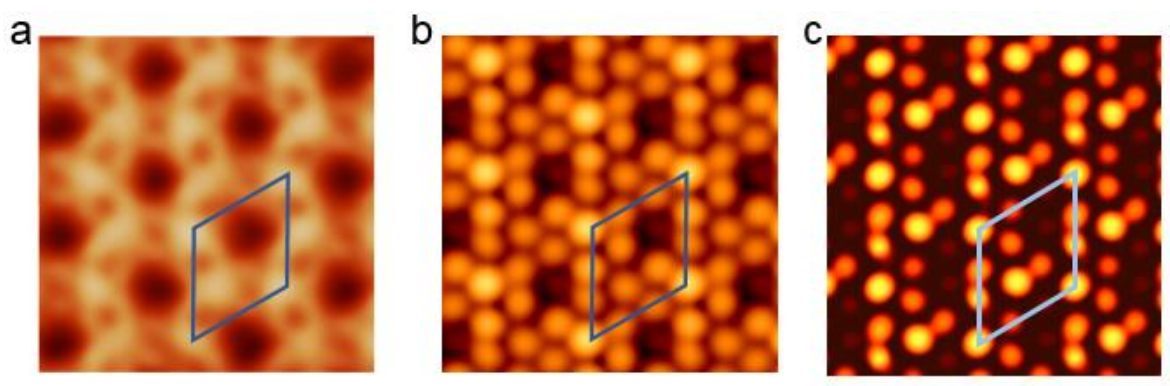

Figure S3 | STM image simulations of 1H-PtSe2 on $\operatorname{Pt}(111)$ with and without the tip effect. a, A zoomed-in STM image. b, A simulated STM image without considering the tip effect. c, A simulated STM image considering the tip effect. As the simplest approximation, we model the tip by a single Se atom, corresponding to the case that a Se atom gets attached to the STM tip. With the tip effect, the spot inside the unit cell has similar brightness as the spots at the corners, in agreement with the data. 
4 The phonon dispersion of a free-standing 1H-PtSe 2 monolayer.

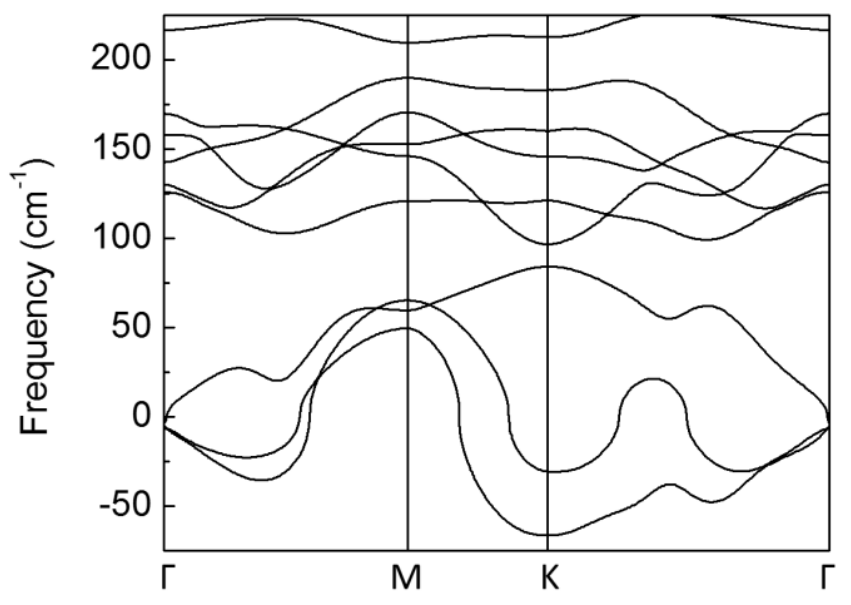

Figure S4 | Phonon dispersion of a free-standing 1H-PtSe 2 monolayer. There are negative-frequency phonon modes indicating the structure is not stable. 


\section{Work function difference between 1H- and 1T- domains.}
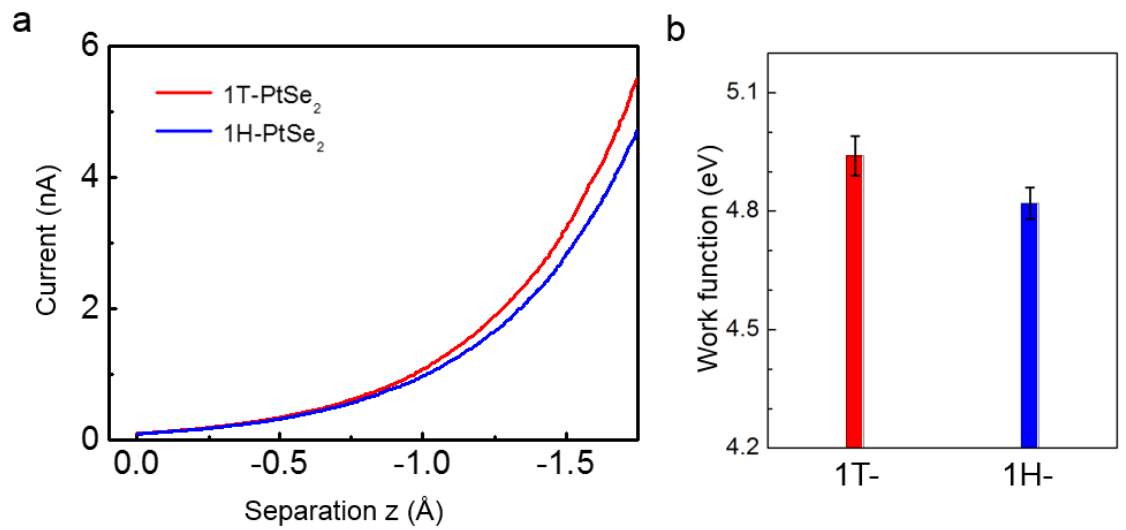

Figure S5 | I(z) spectra and the derived local work function in 1T- and 1H- domains in a patterned 1H/1T-PtSe 2 film. a, Averaged I(z) spectra and $\mathbf{b}$, Derived local work function in 1T- and $1 \mathrm{H}$ - domains. The work function is derived with the method described in Ref. 31. In 1T-domains, the average local work function is $4.94 \pm 0.05 \mathrm{eV}$. In $1 \mathrm{H}$ domains, the average local work function is $4.82 \pm 0.04 \mathrm{eV}$. DFT calculations also reveal a larger work function for 1T-PtSe, $5.21 \mathrm{eV}$, compared with $5.15 \mathrm{eV}$ for $1 \mathrm{H}-\mathrm{PtSe} \mathrm{e}_{2}$. These results provide further evidence of the existence of $1 \mathrm{H}-\mathrm{PtSe}_{2}$. 
6. Atomic structures of 1T-PtSe2 monolayer with $12.5 \%$ Se vacancies.
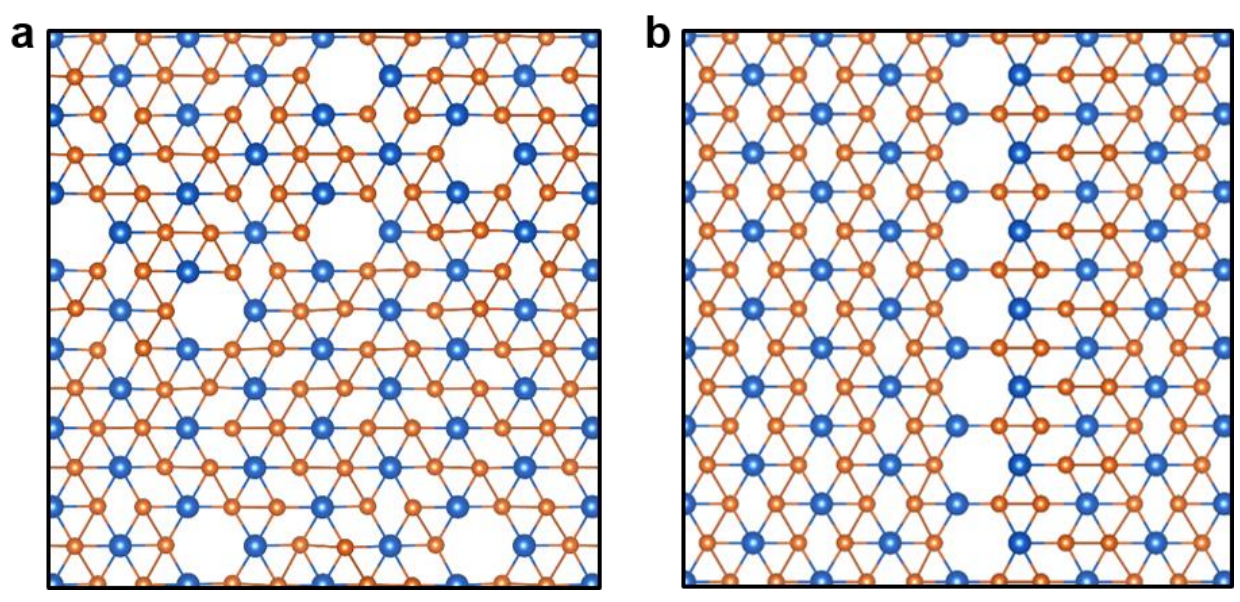

Figure S6 | Atomic structures of 1T-PtSe2 monolayer with 12.5\% Se vacancies. a, Se vacancies are random. $\mathbf{b}$, Se vacancies form lines. DFT calculations indicate that vacancies in lines are more energetically favored by $0.02 \mathrm{eV}$ per Se vacancy. 


\section{Atomic structures of Se vacancies surrounding PtSe2 triangles.}
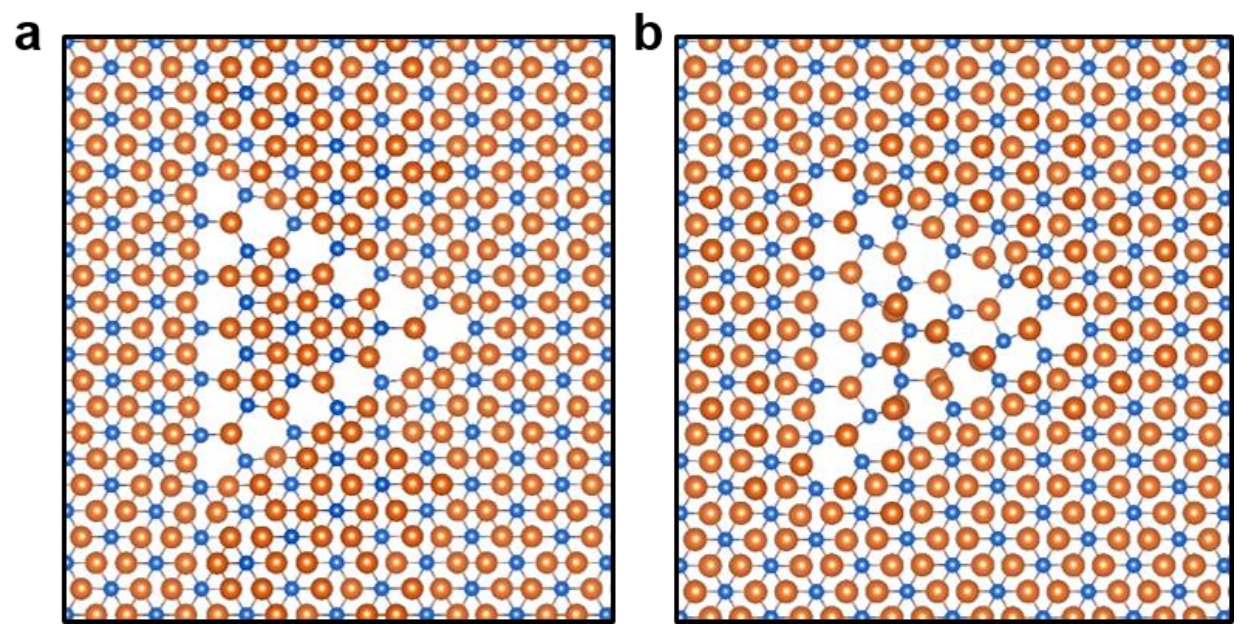

Figure S7 | Atomic structures of Se vacancies surrounding PtSe 2 triangles. a, PtSe 2 triangle in $1 \mathrm{~T}$ phase. $\mathbf{b}, \mathrm{PtSe}_{2}$ triangle in $1 \mathrm{H}$ phase. DFT calculations indicate that $\mathrm{PtSe}_{2}$ triangle in $1 \mathrm{~T}$ phase is more energetically favored by $0.35 \mathrm{eV}$ per Se atom $(0.70 \mathrm{eV}$ per $\mathrm{PtSe}_{2}$ unit). It is worth noticing that in the presence of Se vacancy lines, the energy difference between $1 \mathrm{H}$ - and $1 \mathrm{~T}-\mathrm{PtSe}_{2}$ is significantly lowered. For a perfect $\mathrm{PtSe}_{2}$ monolayer, the energy difference between $1 \mathrm{H}-$ and $1 \mathrm{~T}-\mathrm{PtSe}_{2}$ is calculated to be $1.33 \mathrm{eV}$ per formula unit, while it is only $0.70 \mathrm{eV}$ per formula unit in the presence of Se vacancy lines. The activation energy for the observed transformation should then be $\sim 0.7 \mathrm{eV}$ for each formula unit because the $1 \mathrm{H}$ phase is unstable. It is generally the case that activated processes, e.g., diffusion, occur at room temperature if the activation energy is $\leq 1 \mathrm{eV}$. For example, Ir atoms diffuse on Ir surfaces at room temperature with a migration barrier of $0.7 \mathrm{eV}[32,33]$. Thus, the 1T-to-1H transformation that we observe can indeed occur after annealing at $400{ }^{\circ} \mathrm{C}$. Similar phenomena have been observed in other TMD materials, where thermal annealing at elevated temperature $\left(400{ }^{\circ} \mathrm{C}\right.$ to $\left.700{ }^{\circ} \mathrm{C}\right)$ leads to the formation of triangular inversion domains in $\mathrm{MoS}_{2}$ [34] and 2H-to-1T phase transition in Re-doped $\mathrm{MoS}_{2}$ [21]. 


\section{Produce 1H/1T PtSe 2 triangular pattern with an off-center crucible to control the Se initial density.}

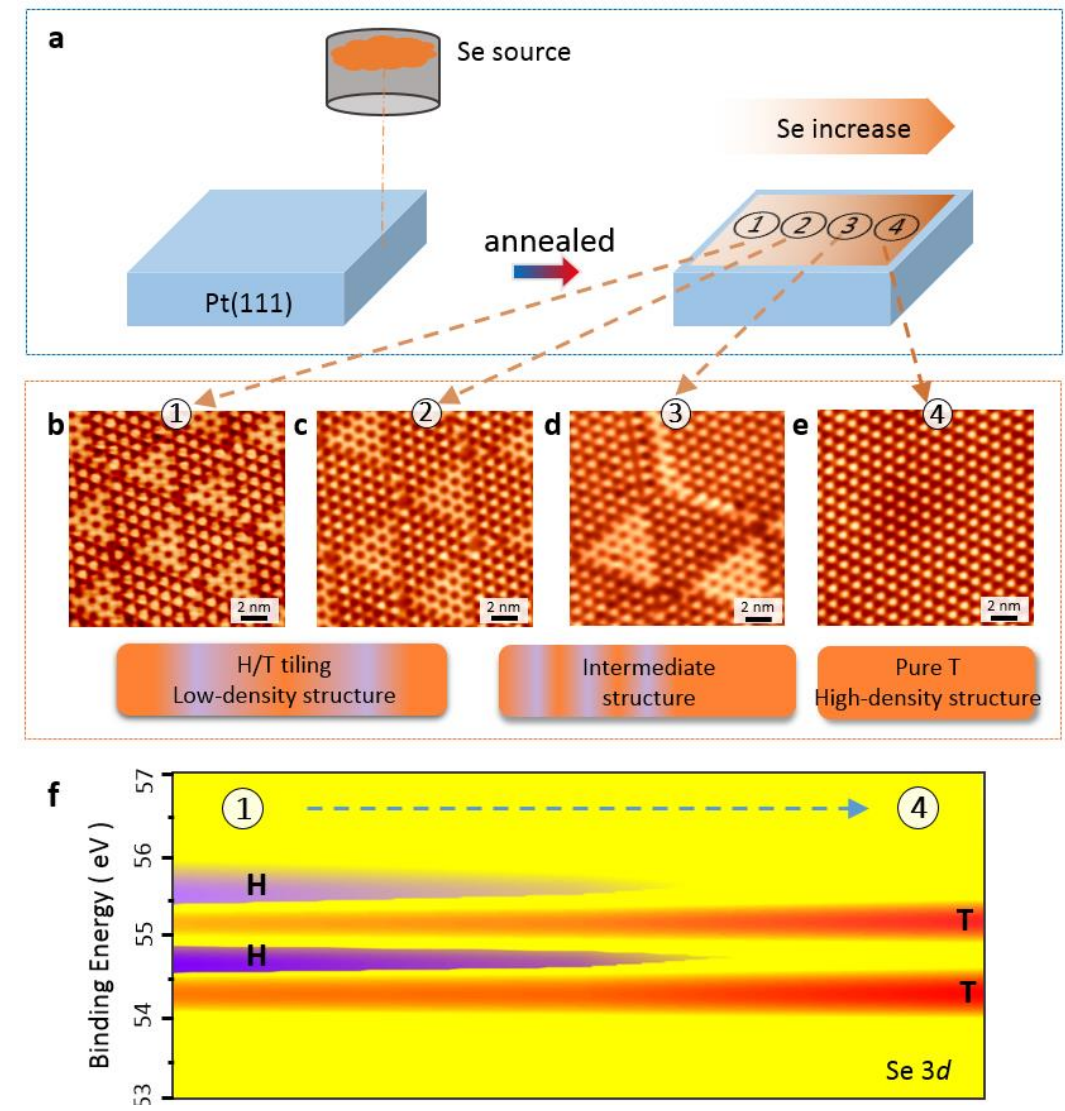

Figure S8 | Produce 1H/1T triangular pattern with an off-center crucible to control the Se initial density. a, Schematic diagram of the experimental method. The crucible filled with Se source is set at the edge instead of the center of substrate. The off-centered deposition leads to a varying initial density of Se atoms at the different positions of the sample. Four different areas with increasing Se density were chosen to investigate the structural variability of $\mathrm{PtSe}_{2}$ films. b, STM image of a triangular tiling patterns of $1 \mathrm{H}$ and $1 \mathrm{~T} \mathrm{PtSe}_{2}$ exist in the area (1) with the relatively low initial Se density. c, d, The number of $1 \mathrm{H}-\mathrm{PtSe}_{2}$ domains decreases as the density of Se atoms increases, demonstrating intermediate structures (2) (3)). e, The continuously increasing Se density finally leads to $1 \mathrm{~T}-\mathrm{PtSe}_{2}$ film (4)). f, The XPS mapping of Se $3 d$ electrons along the direction of increasing Se density areas (area (1) to area (4)) demonstrates a significant decrease of the $1 \mathrm{H}-\mathrm{PtSe}_{2}$ and increase of the $1 \mathrm{~T}-\mathrm{PtSe}_{2}$. For all the STM images, $\mathrm{V}_{\mathrm{s}}=-1.0 \mathrm{~V}, \mathrm{I}_{\mathrm{t}}=0.1 \mathrm{nA}$. 


\section{9. in situ LEED and XPS characterizations of 2D patterned CuSe film.}
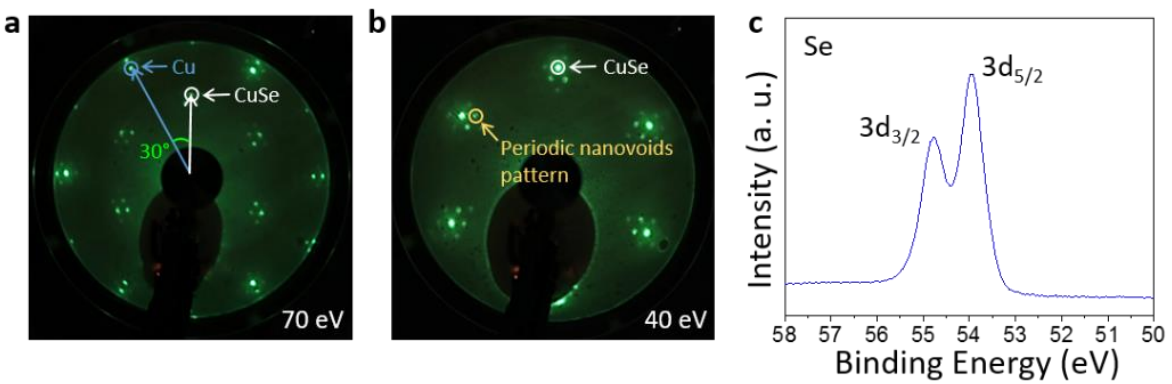

Figure S9 | LEED and XPS characterizations of 2D patterned CuSe film. a, b, Two LEED patterns obtained in the full layer patterned CuSe sample on the $\mathrm{Cu}(111)$ substrate at $70 \mathrm{eV}$ (a) and $40 \mathrm{eV}$ (b), respectively. The outer six diffraction spots in (a) (marked by blue circle) are assigned to the six-fold symmetry of the $\mathrm{Cu}$ substrate and the inner six diffraction spots (marked by white circle) are assigned to the three-fold symmetry of the $\mathrm{CuSe}$ lattice. The figure clearly shows that the angle between the CuSe lattice and the $\mathrm{Cu}$ substrate is $30^{\circ}$, which is in good agreement with the STM data. In addition, the diffraction spots (marked by yellow circle in (b)) surrounding the CuSe diffraction spots are induced by well-defined hexagonal periodic nanopores in the 2D patterned CuSe film observed in the STM images. c, The core level XPS spectrum of patterned CuSe sample on $\mathrm{Cu}(111)$ substrate. Two perfect singlet peaks can be resolved in the Se $3 d$ signal, $54.77 \mathrm{eV}$ and 53.95 $\mathrm{eV}$, which correspond to the binding energy positions of $\mathrm{Se} 3 d_{3 / 2}$ and $3 d_{5 / 2}$ peaks, respectively, indicating the dominance of $\mathrm{Se}^{2-}$ chemical states in the CuSe sample. 
10. Atomic models to explain the formation of 13-atoms-missing pore.
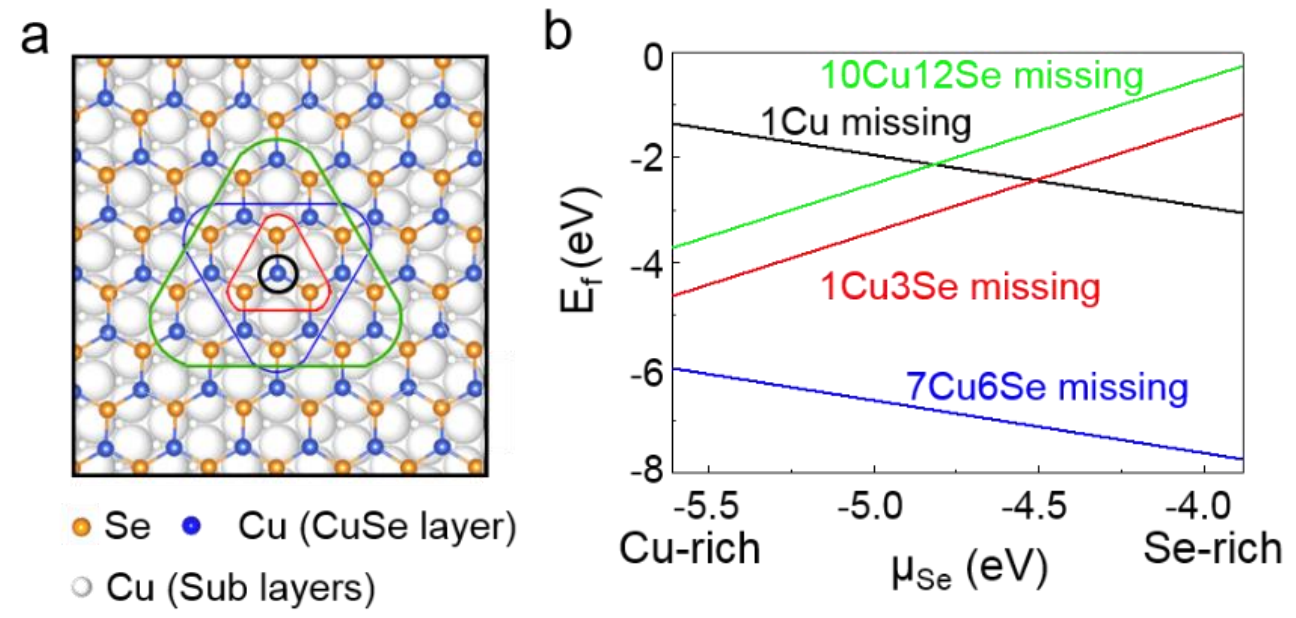

Figure S10 | Formation energies of pores with different sizes in $\mathrm{CuSe} / \mathrm{Cu}(111)$. a, Atomic configurations to show pores with different possible size. Black, red, blue, and green circles correspond to $1 \mathrm{Cu}, 1 \mathrm{Cu} 3 \mathrm{Se}, 7 \mathrm{Cu} 6 \mathrm{Se}$, and 10Cu12Se atoms missing, respectively. b, Formation energy of pores with different sizes. The pore with 13 atoms missing is always energetically favored. Formation energies are negative, corresponding to energy gain. It is clear that the formation of $7 \mathrm{Cu} 6 \mathrm{Se}$ pores is energetically preferred and occurs spontaneously.

The relative stability of nanopores with different size was tested by comparing their formation energies. The formation energy is a function of the chemical potential of Se:

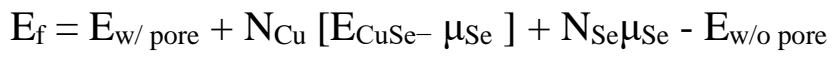

where $E_{w / p o r e}$ and $E_{w / o}$ pore are the total energies with and without pores. $N_{C u}$ is the number of missing $\mathrm{Cu}$ atoms. $\mathrm{N}_{\mathrm{Se}}$ is the number of missing Se atoms. $\mu_{\mathrm{Se}}$ is the chemical potential of Se. For Se-rich conditions, $\mu_{\mathrm{Se}}$, was chosen as the energy of a Se atom in $\mathrm{Se}_{8}$ molecules.

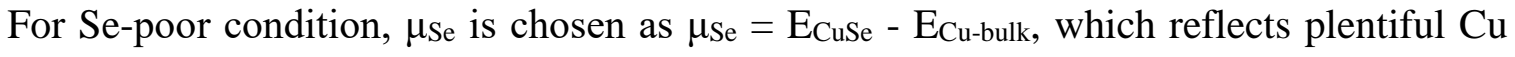
atoms in bulk form. 


\section{Comparison between $13 \mathrm{Fe}$ and $15 \mathrm{Fe}$ atoms in a nanopore in CuSe monolayer.}
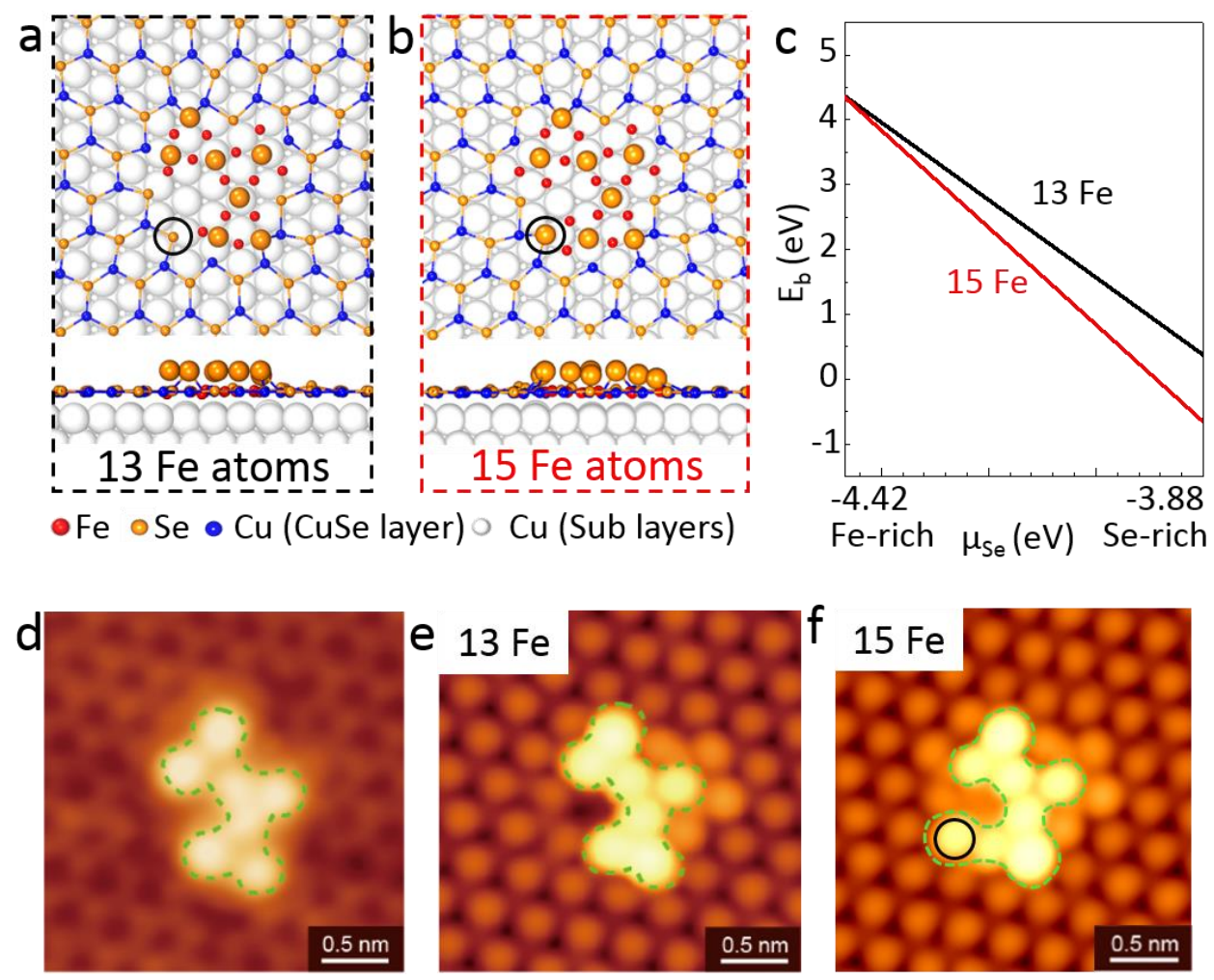

Figure S11 | Comparison between $13 \mathrm{Fe}$ and $15 \mathrm{Fe}$ atoms in a nanopore in CuSe

monolayer. a, b, Top views and side views of atomic models of $\mathrm{Fe}_{\mathrm{x}} \mathrm{Se}_{\mathrm{y}}$ clusters with 13 (a) and $15 \mathrm{Fe}$ atoms (b), respectively. In the 15 atom-configuration, there is one more elevated Se atom (labeled by black circles). c, Binding energy of clusters with 13 and 15 $\mathrm{Fe}$ atoms as function of $\mu_{\mathrm{Se}}$, the chemical potential of Se. $\mu_{\mathrm{Se}}$ is chosen in the range of -4.42 $\mathrm{eV}<\mu_{\mathrm{Se}}<-3.88 \mathrm{eV}$, corresponding to Fe-rich and Se-rich cases, respectively. For virtually all values of $\mu_{\mathrm{Se}}$, clusters with $13 \mathrm{Fe}$ atoms are energetically favored. d, Experimental STM image. e, Simulated image with $13 \mathrm{Fe}$ atoms. f, Simulated image with $15 \mathrm{Fe}$ atoms. There is an extra bright spot for the $15 \mathrm{Fe}$ atoms configuration (labeled by black circle). Note that the elevated (bright) atoms are Se. It is clear that the model with $13 \mathrm{Fe}$ atoms in a pore is in better agreement with the experimental data. 
12. Fe clusters in parallelogram-shape nanopores at domain boundary.

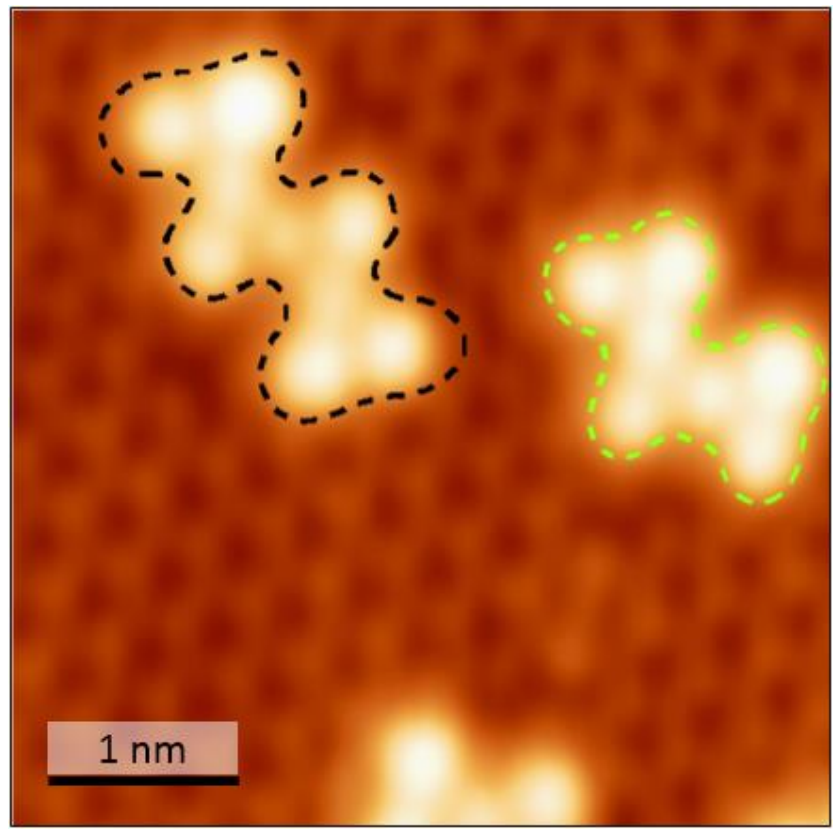

Figure S12 | A high-resolution STM image showing a zigzag-shaped cluster in a parallelogram-shape nanopore at domain boundary (indicated by the black dashed line). For comparison, a W-shaped cluster in a triangular nanopore is also shown (indicated by the green dashed line). The zigzag-shaped cluster can be viewed as an extended Wshaped cluster. $\mathrm{V}_{\mathrm{s}}=-0.1 \mathrm{~V}, \mathrm{I}_{\mathrm{t}}=1.5 \mathrm{nA}$. 
13. Stability tests in ambient conditions.
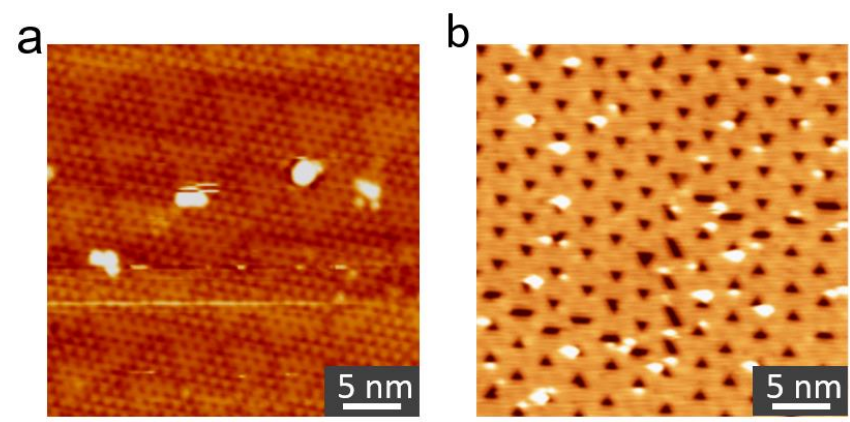

Figure S13 | STM images of intrinsically patterned materials to prove the stability in ambient conditions. a, 1H/1T- $\mathrm{PtSe}_{2}$ on a $\mathrm{Pt}(111)$ substrate. $\mathrm{V}_{\mathrm{s}}=-1.6 \mathrm{~V}, \mathrm{I}_{\mathrm{t}}=0.1 \mathrm{nA}$. b, $\mathrm{CuSe}$ on a $\mathrm{Cu}(111)$ substrate. $\mathrm{V}_{\mathrm{s}}=-1.5 \mathrm{~V}, \mathrm{I}_{\mathrm{t}}=0.1 \mathrm{nA}$. The samples were exposed in air for more than 12 hours, then put back in the high-vacuum chamber and annealed at $200{ }^{\circ} \mathrm{C}$ for $\mathrm{PtSe}_{2}$ and $400{ }^{\circ} \mathrm{C}$ for $\mathrm{CuSe}$ to remove possible adsorbates. The STM images show that, except for some contaminants (big bright spots), the samples keep their patterns.

\section{Adsorption of different molecules on $1 \mathrm{H}$ and $1 \mathrm{~T} \operatorname{PtSe}_{2}$ on $\operatorname{Pt}(111)$.}

Table S1 | Binding energies of different molecules on 1H- and 1T-PtSe2 on $\operatorname{Pt}(111)$ in a unit of $\mathbf{e V}$. Calculation results show that these molecules prefer to adsorb on the $1 \mathrm{H}$ region.

\begin{tabular}{ccc}
\hline & on $1 \mathrm{H}-\mathrm{PtSe}_{2} / \mathrm{Pt}(111)$ & on $1 \mathrm{~T}-\mathrm{PtSe} \mathrm{e}_{2} / \mathrm{Pt}(111)$ \\
\hline $\mathrm{NO}_{2}$ & 1.28 & 0.38 \\
$\mathrm{CO}$ & 0.32 & 0.02 \\
$\mathrm{CO}_{2}$ & 0.20 & 0.05 \\
$\mathrm{C}_{2} \mathrm{H}_{4}$ & 0.46 & 0.18 \\
$\mathrm{C}_{22} \mathrm{H}_{14}$ & 1.31 & 1.00 \\
\hline \hline
\end{tabular}




\section{References:}

31 Zandvliet, H. J. W. \& van Houselt, A. Scanning tunneling spectroscopy. Annu. Rev. Anal. Chem., 2, 37-55 (2009).

32 Barth, J. V., Brune, H., Fischer, B., Weckesser, J. \& Kern, K. Dynamics of surface migration in the weak corrugation regime. Phys. Rev. Lett., 84, 1732 (2010).

33 Chen, C. \& Tsong, T. T. Self-diffusion on the reconstructed and nonreconstructed $\operatorname{Ir}(110)$ surfaces. Phys. Rev. Lett., 66, 1610 (1991).

34 Lin, J., Pantelides, S. T. \& Zhou, W. Vacancy-induced formation and growth of inversion domains in transition-metal dichalcogenide monolayer. ACS Nano, 9, 5189-5197 (2015). 